\title{
Aportes al conocimiento de la ecología de Atelopus balios (Anura: Bufonidae) en el Naranjal, Ecuador
}

Contributions to knowledge of the ecology of Atelopus balios (Anura: Bufonidae) from Naranjal, Ecuador

Pérez-Lara MB, Ramírez-Jaramillo SM. Aportes al conocimiento de la ecología de Atelopus balios (Anura: Bufonidae) en el Naranjal, Ecuador. Rev Colombiana Cienc Anim. Recia. 2021; 13(1):e774. https://doi.org/10.24188/recia.v13.n1.2021.774

Universidad de Sucre, Colombia

Los autores permiten a RECIA reimprimir el material publicado en él. En caso de que un autor quiera traducir o usar una publicación parcial o completa de nuestro Diario, el autor debe obtener un permiso por escrito del editor de la revista.

Copyright (C) 2020. El (los) autor (es). Este es un artículo de acceso abierto distribuido bajo los términos de Creative Commons Attribution 4.0 (https://creativecommons.org/licenses/by-ncsa/4.0/), El uso, distribución o reproducción está permitido, siempre que se acrediten al autor original y al propietario del copyright y que se cite la publicación original en esta revista, de acuerdo con la práctica académica aceptada. No se permite el uso, distribución o reproducción que no cumpla con estos términos. 


\title{
Aportes al conocimiento de la ecología de Atelopus balios (Anura: Bufonidae) en el Naranjal, Ecuador
}

\author{
Contributions to knowledge of the ecology of Atelopus balios (Anura: Bufonidae) from \\ Naranjal, Ecuador
}

María B. Pérez-Lara. Bióloga.

DOI: https://doi.org/10.24188/recia.v13.n1.2021.774

Instituto Nacional de Biodiversidad. División de Herpetología. Rumipamba 341 y Av. de los Shyris, Quito - Ecuador.

Proyecto de "Conservación de Anfibios y Uso Sostenible de Recursos

Genéticos" (PARG), Quito - Ecuador.

maryperez89@hotmail.com

(iD https: //orcid.org/0000-0002-1845-4938

Salomón M. Ramírez-Jaramillo. Biólogo.

Investigador Independiente. Santa Isabel, Quito. Ecuador. Proyecto de

"Conservación de Anfibios y Uso Sostenible de Recursos Genéticos"

(PARG), Quito - Ecuador.

*kp-7sz@hotmail.com, sz.biosupay@gmail.com

Recepción: 15 junio 2020

https://orcid.org/0000-0002-3998-7434

\section{RESUMEN}

Atelopus balios es un sapo endémico de Ecuador, con categoría amenazada debido al cambio de cobertura vegetal y la polución ambiental. Se desconoce sobre sus poblaciones e interacciones ecológicas. El presente estudio da a conocer una línea base sobre el tamaño poblacional y algunos aspectos ecológicos. Durante octubre y noviembre de 2017, se estimó el tamaño poblacional de A. balios en una localidad de Naranjal, piedemonte de la cordillera occidental, Ecuador. Se realizó dos transectos lineales de 1000 metros en dos ríos de la región, donde se hizo dos muestreos diurnos de capturarecaptura por medio de encuentros visuales, y se utilizó la fotoidentificación para estimar el tamaño de la población. También se evaluó su tamaño corporal, estructura poblacional, uso de sustrato, su actividad, y su distribución vertical y horizontal. Obtuvimos 32 registros que corresponden a 29 individuos únicos (T1= 3 ind y T2= 26 ind). La proporción de machos/hembras fue de 8.6:1. En T1 no se pudo aplicar el estimador de población. En T2 se estimó 52.25£EE18.64 individuos. El 59.37\% utiliza el sustrato de hoja, el 75\% presentó actividad desplazándose al momento de la captura, su distribución vertical es entre 20 y $250 \mathrm{~cm}$ de alto, su distribución horizontal fue entre 20 y $1000 \mathrm{~cm}$. Se desconoce sobre la presencia de quitridio, pero se considera importante diagnosticar su presencia y prevalencia. Aunque la abundancia en ambos transectos varía, se considera que las poblaciones son estables y su fluctuación es normal. Por lo que es importante realizar monitoreos.

Palabras clave: Conservación; distribución vertical y horizontal; tamaño y estructura poblacional; sapo Jambato; sustrato.

\begin{abstract}
Atelopus balios is a toad endemic to Ecuador, with a threatened category due to change in plant cover and environmental pollution. It is unknown about their populations and ecological interactions. This study discloses a baseline on population size and some ecological aspects. During October and November 2017, the population size of A. balios was estimated in a locality of Naranjal, foothills of the western mountain range, Ecuador. Two linear transects of 1000 meters were carried out in two rivers of the region, where two daytime sampling of capture-recapture was performed through visual encounter surveys, and photoidentification was used to estimate population size. Also there was evaluated its corporal size, population structure, used substrate, its activity, and its vertical and horizontal distribution. We obtained 32 records that correspond to 29 only individuals (T1 = 3 ind and T2 = 26 ind). The male/female ratio was 8.6:1. In T1, the population

Como citar (Vancouver).

Pérez-Lara MB, Ramírez-Jaramillo SM. Aportes al conocimiento de la ecología de Atelopus balios (Anura: Bufonidae) en el Naranjal, Ecuador. Rev Colombiana Cienc Anim. Recia. 2021; 13(1):e774. https://doi.org/10.24188/recia.v13.n1.2021.774
\end{abstract}


estimator could not be applied. In T2, 52.25 EEE18.64 individuals were estimated. We found that 59.37\% used leaf substrate, $75 \%$ had activity moving at the time of capture, its vertical distribution is between 20 and $250 \mathrm{~cm}$ high, its horizontal distribution was between 20 and $1000 \mathrm{~cm}$. It is unknown about the presence of quitridium, but it is considered important to diagnose its presence and prevalence. Although the abundance in both transects varies, populations are considered stable and their fluctuation is normal. So it's important to perform monitoring.

Keywords: Conservation; Jambato toad; population size and structure; substrate; vertical and horizontal distribution.

\section{INTRODUCCIÓN}

El género Atelopus (Dumérily Bibron, 1841), es un grupo de ranas que incluye un total de 96 especies descritas formalmente, que se distribuyen en distintos ecosistemas de la región Neotropical (1). De acuerdo con la Unión Internacional para la Conservación de la Naturaleza-UICN (2), en Ecuador el género está representado por 25 especies, de las cuales 18 especies (72\%) son consideradas endémicas. La situación actual de conservación y el grado de amenazas son preocupantes, ya que el $84 \%$ de los Atelopus ecuatorianos se incluyen en alguna categoría de amenaza, causadas principalmente por la reducción de su hábitat, el cambio climático y enfermedades $(2,3)$.

Las disminuciones poblacionales que se ha producido en muchas especies de anfibios durante las últimas décadas, y en especial sobre este género de ranas, es un fenómeno global reportado desde finales de la década de 1980 hasta la actualidad $(3,4)$; y dada su relevancia e impacto, se ha propiciado varias investigaciones e iniciativas de conservación enfocadas en conocer las causas de esta problemática y responder a las amenazas (5).

Según la UICN (2), Atelopus balios es considerada como una especie en Peligro Crítico (CR) bajo los criterios B1ab (i, iii), ya que su área de distribución se restringe a $55 \mathrm{~km}^{2}$, y es conocida únicamente en cuatro localidades en los bosques húmedos tropicales occidentales en las provincias de Azuay, Cañar y Guayas en Ecuador, con distribución desde 100 a 900 metros de altitud. La mayor amenaza en la actualidad es la agricultura, ganadería, minería y la polución ambiental. Los estudios sobre la biología y conservación de la especie presentan importantes vacíos de información como: 1) existencia de otras poblaciones; 2) efectos de los factores de presión antrópica sobre las poblaciones; 3) presencia de quitridiomicosis y otros patógenos; 4) falta de un conocimiento más preciso de las interrelaciones con el ambiente que ocupa la especie. Con estos antecedentes, resulta imprescindible implementar acciones urgentes destinadas a conservar las poblaciones conocidas en el área de distribución de la especie.

En este contexto, el monitoreo biológico se presenta como una herramienta de suma importancia para los estudios de ecología, conservación y manejo de vida silvestre, ya que, determinar posibles cambios temporales-espaciales ocurridos en la dinámica de las poblaciones, puede resultar clave para la comprensión del efecto de sus posibles causas naturales o de origen antrópico sobre las especie $(6,7)$. De este modo, el proyecto "Conservación de Anfibios y Uso Sostenible de Recursos Genéticos (PARG)", reconoce a Atelopus balios como una de las especies objetivo de conservación in situ y ex situ en Ecuador.

En este estudio se presenta información de línea base fundamentada en dos muestreos de captura-recaptura, cuyo objetivo es sumar información valiosa sobre el tamaño de la población de A. balios, su tamaño corporal, estructura de la población, el uso de sustrato, su actividad, y su distribución vertical y horizontal en el área protegida Cerro “Las Hayas” y zona adyacente, considerada una localidad clave para la conservación de la especie.

\section{MATERIALES Y MÉTODOS}

Área de estudio. El estudio se llevó a cabo en la comunidad 23 de noviembre que se ubica en la provincia del Guayas, Cantón Naranjal, Parroquia Naranjal (0243’ S; 79³8’ W), comprende una extensión de aproximadamente 650 ha. Dentro de la comunidad se encuentra el Área Provincial Natural de Recreación (APNR) Cerro Las Hayas con una superficie de 397.16 ha (8), localizada en el piedemonte de la cordillera entre los 60 y 640 msnm (Figura 1). En dirección NO-SE transcurren dos ríos principales casi paralelos y distanciados por $1.2 \mathrm{~km}$ entre sí, con anchos entre 6 y $10 \mathrm{~m}$. Ambos ríos sustentan a la comunidad 23 de noviembre, y posteriormente desembocan en el Océano Pacífico, poseen remanentes de bosque rodeado de áreas de cultivos como plátano, cacao, y pastizales. 


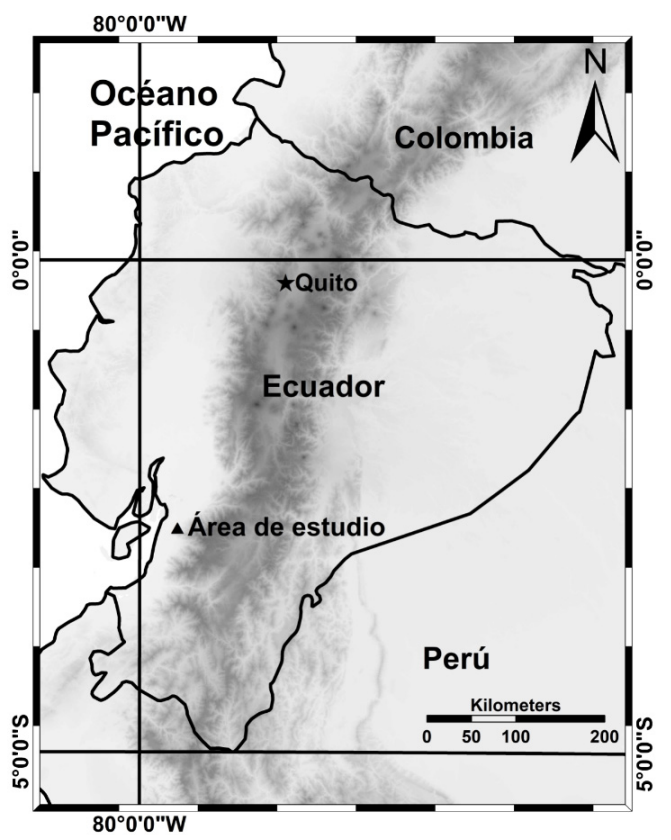

Figura 1. Ubicación del área de estudio en el Naranjal.

El río Mina presenta topografía de colinas con pendientes entre $5^{\circ}$ a $>50^{\circ}$ en algunos tramos, y se ubica en el ecosistema de bosque siempreverde estacional pie montano de la Cordillera Occidental de los Andes (9). Las laderas escarpadas presentan vegetación secundaria y arbustiva, con árboles entre 20 y $25 \mathrm{~m}$ de alto. Las orillas poseen sustrato de fondo pedregoso y grandes piedras, no se observa acumulación de material vegetal en descomposición.

El río Ochoa presenta topografía con pendiente $<10^{\circ}$. Se ubica en el ecosistema de Bosque semideciduo de tierras bajas del Jama Zapotillo (9), desde 60 hasta 300 msnm. Presenta vegetación secundaria circundante y sotobosque en gradiente de moderado a abierto, con árboles entre 20 y $25 \mathrm{~m}$ de alto. Las orillas del río presentan sustrato de fondo pedregoso y grandes piedras, y poca materia orgánica.

Método. Durante octubre y noviembre de 2017, siguiendo el cauce en dos ríos de la localidad, se estableció en cada uno un transecto lineal de $1000 \mathrm{~m}$ de distancia y ancho variado entre 5-10 m, donde se realizaron relevamientos visuales para obtener datos de abundancia sobre la especie $(7,10)$. Así: transecto río Mina (T1) dentro del APNR Cerro Las Hayas, y transecto río Ochoa (T2) aledaño al centro de la comunidad (Figura 1). En cada transecto se emplearon tres días seguidos de muestreo efectivo que fue realizado por dos personas (técnico y guía de la comunidad) con un esfuerzo de muestreo diario de 6 horas/persona, acumulando así un total de 36 horas de muestreo para cada transecto. Para evitar el conteo repetido del mismo individuo se utilizó la técnica de fotoidentificación, fotografiando el dorso de cada individuo capturado y asignándole un código único $(10,11)$. Se registró, además, el sexo (definido por diferencias de tamaño corporal o evidencias de la presencia de ovocitos en la cavidad corporal), actividad al momento de captura (desplazándose, perchando), sustrato (hoja, rama, roca), la Longitud Rostro Cloaca (LRC), posición vertical en relación al suelo (altura en cm desde el suelo), posición horizontal (distancia de ubicación hacia el borde del río en cm) (7,10,11).

Para estimar el tamaño de la población se utilizó el estimador de Bailey incluyendo el error estándar (EE) para dos muestras de captura y recaptura (12). La estructura poblacional de machos y hembras, la LRC, el uso de sustrato y su distribución vertical y horizontal fueron analizadas mediante estadística descriptiva con el programa estadístico BioEstat 5.0 (13).

\section{RESULTADOS}

Se obtuvo un total de 32 registros (tres en T1 río Mina y 29 en T2 río Ochoa) (Tabla 1). En T1 río Mina no se pudo aplicar el estimador de población por tener muy pocos datos. En T2 río Ochoa se estimó 52.25£EE18.64 individuos. Se obtuvo el registro de 29 individuos únicos (tres en T1 y 26 en T2) (Figura 2). En ambos transectos se registró 26 machos y tres hembras, para una proporción de sexo de 8.6:1. En T1 únicamente se registraron machos; mientras que en T2 se registraron 23 machos y 3 hembras, para una proporción de sexo de 7.6:1. El sustrato más frecuentado de $A$. balios fue hoja con el 59.4\%. La actividad frecuente al momento de la captura fue desplazándose con el 75\% (Tabla 1). 
Tabla 1. Estructura poblacional, tipo de sustrato y actividad ocurridos en T1= Río Mina y T2= Río Ochoa.

\begin{tabular}{cccccc}
\hline Ítem de análisis & Categorías establecidas & T1 & T2 & Total & $\overline{\mathrm{X}} \pm \mathbf{D S}$ \\
\hline \multirow{2}{*}{ Estructura poblacional } & Machos & 3 & 26 & 29 & $16 \pm 18.38$ \\
& Hembras & 0 & 3 & 3 & $\mathrm{CV}: 114.9 \%$ \\
Tipo de sustrato & Hoja & 2 & 17 & 19 & \\
& Rama & 1 & 9 & 10 & $10.67 \pm 8.02$ \\
Rctividad & Roca & 0 & 3 & 3 & $\mathrm{CV}: 75.20 \%$ \\
& Desplazándose & 1 & 23 & 24 & $16 \pm 11.31$ \\
& Perchando & 2 & 6 & 8 & $\mathrm{CV}: 70.71 \%$ \\
& Total & 3 & 29 & 32 & \\
\hline
\end{tabular}

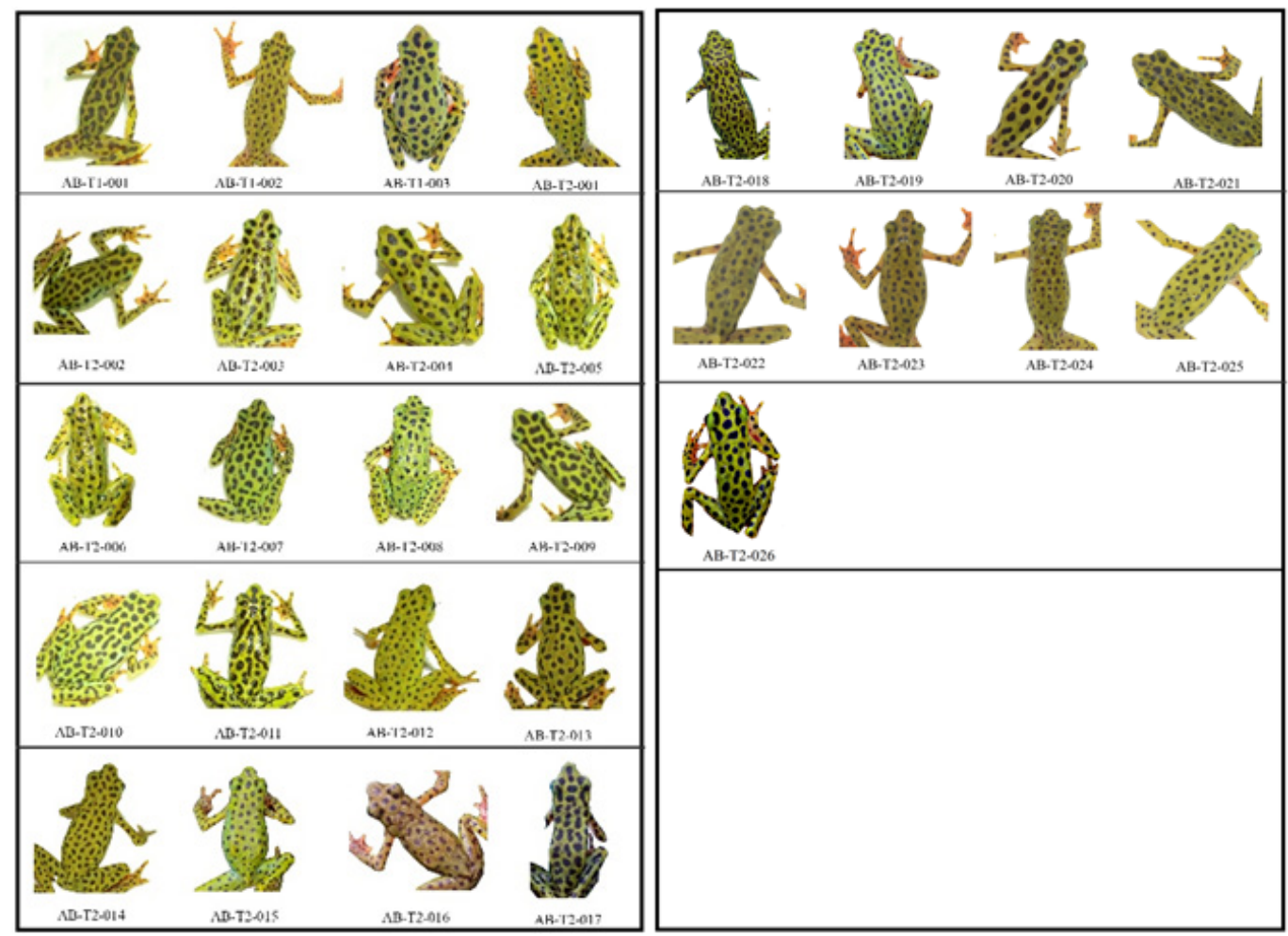

Figura 2. Láminas que muestran los dorsos de 29 individuos de A. balios con su código de registro en el área de estudio.

La LRC varío entre 20.1 y $35.4 \mathrm{~mm}(\bar{X}=24.66 \pm 3.55 \mathrm{~mm})$, entre 20.1 y $27.96 \mathrm{~mm}$ para machos y entre 30.1 y $35.4 \mathrm{~mm}$ para hembras. La distribución vertical fue entre 20 y $250 \mathrm{~cm}$ de altura $(\bar{X}=127.96 \pm 64.6 \mathrm{~cm})$. En T1 fue entre 30 y 120 $\mathrm{cm}(\bar{X}=90 \pm 51.96 \mathrm{~cm})$. En T2 fue entre 20 y $250 \mathrm{~cm}(\bar{X}=131.89 \pm 65.25 \mathrm{~cm})$. La distribución horizontal fue entre 20 y $1000 \mathrm{~cm}(\bar{X}=275.06 \pm 225.32 \mathrm{~cm})$. En T1 fue entre 100 y $300 \mathrm{~cm}(\bar{X}=183.3 \pm 104.08 \mathrm{~cm})$. En T2 fue entre 20 y $1000 \mathrm{~cm}$ $(\bar{X}=284.55 \pm 233.3 \mathrm{~cm})$.

\section{DISCUSIÓN}

En la población estudiada de Atelopus balios la proporción de machos/hembras fue de 8.6:1. Siendo similar a lo reportado en Atelopus varius, donde se registró una proporción de 8.08:1 (14). Pero difieren de lo registrado en Atelopus spumarius donde fue de 4.1:1 (15), en Atelopus zeteki que fue de 2.24:1 (14), en Atelopus cruciger que fue de 2:1 (16), y en contraste para Atelopus laetissimus que fue de 24.5:1 (17). 
Mediante dos muestreos de captura-recaptura, los individuos registrados mediante fotoidentificación mostraron patrones de manchas únicos (Figura 2), señalando así la importancia de este uso de métodos no invasivos de marcaje para especies amenazadas $(10,11)$. El tamaño poblacional estimado en T2, podría sugerir que la población es estable dentro de un área que no se encuentra protegida, y que podría ser anexada a la APNR debido a que su población es más abundante que en T1.

Granda-Rodríguez et al (17), encuentra en Atelopus laetissimus que durante el día prefieren el sustrato de rocas y hojarasca, mientras que su distribución vertical es entre 40 y $120 \mathrm{~cm}$, y la horizontal es a menos de $5 \mathrm{~m}$. El uso de sustrato en A. balios tuvo mayor frecuencia en hoja, luego en rama y en menor proporción en roca (Tabla 1), pero no fue registrado en hojarasca, su distribución vertical tuvo un promedio de $128 \mathrm{~cm}$ de altura, lo cual estaría acorde a la preferencia de sustrato, tan solo el $22 \%$ fue registrado a menos de $50 \mathrm{~cm}$ y coincide con el uso de sustrato en roca. Su distribución horizontal tuvo un promedio de $275 \mathrm{~cm}$ del borde del río, y se encontró que el 78\% (incluido las tres hembras registradas) fue registrado a distancias de más de un metro, lo cual puede cambiar dependiendo de su época reproductiva al igual que en otros Atelopus (17). Esto coincide con el bajo registro de hembras, señalando así que en estos meses de muestreo no es su época reproductiva, y estarían descartados para futuros muestreos donde se busquen hembras para programas de conservación in situ o ex situ. Del mismo en su actividad no se registraron amplexus, lo cual confirmaría este hecho.

Hasta febrero de 2018, se desconoce sobre la presencia de quitridiomicosis, sin embargo, los individuos registrados no presentaban decaimiento y se veían sanos. No obstante, sería importante diagnosticar la presencia y prevalencia de enfermedades.

En cuanto a la diferente abundancia de individuos entre T1 y T2, se desconoce su razón. Se habría esperado que existan más individuos en T1 ya que está dentro de un área protegida, sin embargo, ocurrió lo contrario. Dados estos resultados de escasa abundancia en T1, no se pueden sugerir claras diferencias, sin embargo, se espera que las poblaciones de $A$. balios permanezcan estables y que esta diferencia entre ambos sitios de muestreo sea debida a una fluctuación normal dentro de la población. Por lo cual es importante su monitoreo. Gómez-Hoyos et al. (18), recomienda dos muestreos anuales durante tres años para detectar cambios poblacionales. Es importante señalar que, aunque el presente estudio tiene poca representatividad de muestreo, aporta con información primordial para futuros estudios y programas de conservación.

\section{Conflicto de intereses}

Los autores declaramos que no existe conflicto de intereses entre nosotros o con terceros.

\section{Financiación}

Programa de las Naciones Unidas para el Desarrollo (PNUD). https://www.ec.undp.org/content/ecuador/es/home/ projects/conservacion-de-anfibios-ecuat.html

\section{Agradecimientos}

A L. Coloma, M. Ortega, P. Larco y el Centro Jambatu quienes desarrollaron y coordinaron el Proyecto PARG. A la Comunidad 23 de Noviembre y de Las Hayas por el acceso a predios y grata compañía. Al Ministerio del Ambiente por los permisos de investigación. A M. Vaira por su revisión. A nuestras familias.

\section{REFERENCIAS}

1. Frost DR. Amphibian Species of the World 6.0, an Online Reference. Version 6.0. American Museum of Natural History, New York, USA; 2020 [citado 6 jun 2020]. URL Disponible en: http://research.amnh.org/herpetology/amphibia/ index.html

2. IUCN. The IUCN Red List of Threatened Species. Version 2020-1; 2020 [citado 19 mar 2020]. URL Disponible en: https://www.iucnredlist.org

3. Cohen JM, Civitello DJ, Venesky MD, McMahon TA, Rohr JR. An interaction between climate change and infectious disease drove widespread amphibian declines. Glob Change Biol. 2018; 25:927-937. https://doi.org/10.1111/ gcb.14489 
4. Gómez-Hoyos DA, Cardona W, González-Durán GA, Flechas SV, Kattan GH, Velásco JA. The population decline of Atelopus quimbaya (Anura: Bufonidae) in the Andes of Colombia. Rev Latinoam Herpetol. 2018; 1: 34-42. https:// herpetologia.fciencias.unam.mx/index.php/revista/article/view/11

5. Lewis CHR, Richards-Zawacki CL, Ibáñez R, Luedtke J, Voyles J, Houser P, et al. Conserving Panamanian harlequin frogs by integrating captive-breeding and research programs. Biol Conserv. 2019; 236:180-187. https://doi. org/10.1016/j.biocon.2019.05.029

6. Magnusson W, Braga-Neto R, Pezzini F, et al. Biodiversidade e monitoramento ambiental integrado (Biodiversity and integrated environmental monitoring). Brasil: Áttema Editorial; 2013.

7. Gallina S, López-González C, editores. Manual de Técnicas para el Estudio de la Fauna. Volúmen I. México: Universidad Autónoma de Querétaro-Instituto de Ecología. 2011. https://es.scribd.com/document/217173516/Gallina-SoniaManual-de-Tecnicas-Para-El-Estudio-de-La-Fauna

8. Carvajal R, Camba M, Guillén W, et al. Levantamiento Preliminar de Información de Campo de los remanentes Boscosos de los predios de los hermanos Roberto, Jorge y Carlos Rodríguez Cucalón, Cerro de Hayas, Cantón Naranjal. Ecuador: Informe Técnico Externo; 2013.

9. Ministerio del Ambiente del Ecuador (MAE). Sistema de Clasificación de los Ecosistemas del Ecuador Continental. Ecuador: Subsecretaría de Patrimonio Natural; 2013. https://www.ambiente.gob.ec/wp-content/uploads/ downloads/2012/09/LEYENDA-ECOSISTEMAS ECUADOR 2.pdf

10. Heyer WR, Donnelly MA, McDiarmid RW, et al, editores. Measuring and Monitoring Biological Diversity, standard methods for Amphibians. USA: Smithsonian Institution Press; 1994.

11. Ramírez S, Rodríguez M. Estado poblacional y relaciones ecológicas de Gastrotheca riobambae (Anura: Hemiphractidae) en dos localidades del Volcán Pasochoa. Prov. Pichincha. Ecuador. Serie Zoológica. 2011; 10:69-97. https://journal.espe.edu.ec/ojs/index.php/revista-serie-zoologica/article/view/1449

12. Donnelly MA, Guyer C. Estimating population size. En: Heyer WR, Donnelly MA, McDiarmid RW, et al, editores. Measuring and Monitoring Biological Diversity, standard methods for Amphibians. USA: Smithsonian Institution Press; 1994.

13. Ayres M, Ayres JrM, Ayres DL, et al. BioEstat 5.0: Aplicações Estatísticas nas áreas das Ciências Bio-Médicas. Brasil: Sociedade Civil Mamirauá; 2007.

14. McCaffery R, Richards-Zawacki CL, Lips KR. The demography of Atelopus decline: Harlequin frog survival and abundance in central Panama prior to and during a disease outbreak. Global Ecol Conserv. 2015; 4:232-242. https:// doi.org/10.1016/j.gecco.2015.07.003

15. Tarvin RD, Peña P, Ron SR. Changes in population size and survival in Atelopus spumarius (Anura: Bufonidae) are not correlated with chytrid prevalence. J Herp. 2014; 48(3):291-297. http://dx.doi.org/10.1670/11-269

16. Lampo M, Señaris C, García CZ. Population dynamics of the critically endangered toad Atelopus cruciger and the fungal disease chytridiomycosis. PloS One. 2017; 12(6):1-18. https://doi.org/10.1371/journal.pone.0179007

18. Granda-Rodríguez HD, del Portillo-Mozo A, Renjifo JM. Uso de hábitat en Atelopus laetissimus (Anura: Bufonidae) en una localidad de la Sierra Nevada de Santa Marta, Colombia. Herpetotrópicos. 2008; 4:87-93.

19. Gómez-Hoyos DA, Bolívar-G W, Burbano-Yandi CE, García JL. Evaluación poblacional y estrategia de monitoreo para Atelopus spurrelli en el Parque Nacional Natural Utría, Colombia. Rev Biodiv Neotrop. 2014; 4(2):104-112. http:// dx.doi.org/10.18636/bioneotropical.v4i2.136 